\title{
On the gravity dual of strongly coupled charged plasma
}

\author{
Grzegorz Plewa $^{a}$ and Michat Spaliński ${ }^{a, b}$ \\ ${ }^{a}$ National Center for Nuclear Research, \\ ul. Hoża 69, 00-681 Warsaw, Poland \\ ${ }^{b}$ Physics Department, University of Biatystok, \\ ul. Lipowa 41, 15-424 Biatystok, Poland \\ E-mail: g.plewa@ipj.gov.pl, mspal@fuw.edu.pl
}

ABSTRACT: Locally asymptotically AdS solutions of Einstein equations coupled with a vector field with a weakly curved boundary metric are found within the fluid-gravity gradient expansion up to second order in gradients. This geometry is dual to $1+3$ dimensional hydrodynamics with a conserved current in a weakly curved background. The causal structure of the bulk geometry is determined and it is shown that the black brane singularity is shielded by an event horizon.

KEYWORDS: Gauge-gravity correspondence, Holography and quark-gluon plasmas, Black Holes in String Theory 


\section{Contents}

1 Introduction $\quad 1$

2 The bulk theory, black branes and thermodynamics 3

3 General form of the solution $\quad 5$

3.1 The gradient expansion 5

$\begin{array}{lll}3.2 & \text { Weyl covariance } & 6\end{array}$

$\begin{array}{lll}4 & \text { The solution order by order } & 7\end{array}$

4.1 Zeroth order $\quad 7$

4.2 First order 8

$\begin{array}{lll}4.3 & \text { Second order } & 10\end{array}$

$\begin{array}{llr}5 & \text { Holography } & 11\end{array}$

6 Causal structure of the dual geometry 13

$\begin{array}{lll}7 & \text { Conclusions } & 15\end{array}$

A Second order functions $\quad \mathbf{1 5}$

$\begin{array}{lll}\text { A.1 Scalar sector } & 15\end{array}$

$\begin{array}{ll}\text { A.2 Vector sector } & 18\end{array}$

$\begin{array}{lll}\text { A.3 Tensor sector } & 19\end{array}$

$\begin{array}{ll}\text { B Weyl weights } & 20\end{array}$

$\begin{array}{ll}\text { C Inner horizon at second order } & 20\end{array}$

\section{Introduction}

Applications of the AdS/CFT correspondence to non-static geometries continue to be an active area of research. This is motivated partly be the inherent interest in non-equilibrium processes in strongly coupled quantum theories and partly by pressing questions arising in this context in connection with the phenomenology of heavy ion collisions [1].

An important connection between slowly evolving geometries on the gravity side and hydrodynamic states of $\mathrm{N}=4$ supersymmetric Yang-Mills theory was uncovered in [2] following the earlier observations of [3]. The simplest case of this connection involves only the metric in the bulk, and describes the hydrodynamics of a fluid without any conserved currents beyond the energy-momentum tensor. This so-called fluid-gravity correspondence was subsequently generalized in various directions [4-7]. 
A very important case is that of hydrodynamics with conserved charges. Apart from purely theoretical interest, the motivation for this arises in the context of applications of relativistic hydrodynamics to the evolution of quark-gluon plasma created in heavy ion collisions. In that case the conserved charge is baryon number. ${ }^{1}$ More generally, one is interested in the properties of gauge theory plasma at finite temperature and chemical potential. Such a system is dual to gravity interacting with a vector field. The generalization of fluid-gravity duality to this situation was taken up in $[4,5]$, where a solution of the dual gravitational theory was described (up to second order in the gradient expansion) and transport coefficients of the charge-bearing plasma were calculated (see also $[8,9]$ ). Since the bulk gravity theory as it appears in a string compactification [10] includes a ChernSimons term, the dual hydrodynamics includes terms unexpected from the point of view of classical expositions of the subject. This has lead to very interesting developments which clarify the effects of anomalies in hydrodynamics [11].

The major goal of this article was to determine the causal structure of the dual spacetime. This is motivated by two issues. First of all, the gravitational solution found in the gradient expansion is smooth apart from a singularity at $r=0$ (the black brane singularity). On general grounds it is expected that this singularity should shielded by an event horizon. Establishing this is important and nontrivial. The second issue is that of hydrodynamic entropy currents [12-14]. The event horizon defines an entropy current and the condition that its divergence be non-negative imposes constraints on the transport coefficients. ${ }^{2}$ It is natural to also consider entropy currents defined by dynamical horizons in the geometry [17-19]. This subject will be taken up elsewhere [20].

Much of this article is devoted to establishing the dual solution itself. Compared to the earlier works the calculation presented here differs in a number of ways. First of all, the results generalize the findings of $[4,5]$ in that an arbitrary weakly curved boundary metric is admitted. As in the case without charge, the result can not be obtained just by covariantizing the flat-boundary result, since there is an additional term which involves the curvature of the boundary metric [6]. More importantly from the point of view of locating the event horizon, the form of the solution obtained here is somewhat simpler and more explicit than the earlier results. This is due to a number of factors. Since one of the goals of this study was to determine the causal structure of the dual spacetime geometry, it was convenient to use a parameterization of the zeroth-order solution such that the event horizon at that order can be expressed in a simple way. Such a parameterization (found in [21]) also simplifies the gradient expansion, and especially the perturbative determination of the event horizon of the slowly evolving geometry. Another technical difference is that the present paper makes use of a different gauge than that used in $[4,5]$, following the choice made in $[6,7]$. The advantage of this gauge, apart from simplicity, is that ingoing null geodesics are simply curves of constant boundary coordinate $x$. This defines a natural bulk-boundary map, which is an important element of the holographic construction of the hydrodynamic entropy current. Finally, explicit Weyl covariance in maintained throughout

\footnotetext{
${ }^{1}$ In general one may also consider additional conserved charges, such as strangeness (as long as weak interactions are negligible).

${ }^{2}$ See however $[15,16]$.
} 
(as in $[6,7]$ ), which simplifies the calculations as well as the form of the final results. It turns out, that taken together these simplifications make it possible to write completely explicit formulae for the metric and gauge field, which are somewhat more complicated than those given in [7] for the case of uncharged plasma, but not significantly so. In particular, it is manifest that the results given here reduce to those of [7] in the limit of vanishing charge. Following the standard procedure of holographic renormalization simple and complete expressions for the transport coefficients are also obtained.

A very natural generalization of the computations presented here would be to include a background gauge field (corresponding to background electric and magnetic fields). This would lead to additional transport coefficients. Calculations of this type have been done to first order in the gradient expansion [8, 22, 23], leading to results for the electrical conductivity, for example. In the present study the extension to non-vanishing background fields was not made, although carrying out such computations to second order is feasible. The second order calculations described below are partly motivated by the fact that due to conformal symmetry the differences between the event and apparent horizons only arise at that order. While dynamical horizons are not considered in this article, the methods used here could be used to find the apparent horizon for the geometry under consideration,

The structure of this article is as follows. Section 2 reviews the relevant static gravity solution and some aspects of its thermodynamics. Section 3 describes the general form of the solution and an overview of the computation. Section 4 presents the solution up to second order in the gradient expansion. Section 5 describes the results of the holographic renormalization procedure and the results for the transport coefficients. The causal structure of the geometry is studied in section 6 , where the event horizons are located. Some closing remarks follow in section 7 .

\section{The bulk theory, black branes and thermodynamics}

The action of the five-dimensional Einstein-Maxwell theory under consideration reads

$$
S=\frac{1}{16 \pi G_{N}} \int d^{5} x \sqrt{-g}\left(\frac{12}{L^{2}}+R-F^{2}-\frac{4 \kappa}{3} \epsilon^{A B C D E} A_{A} F_{B C} F_{D E}\right)
$$

where the cosmological constant is denoted by $12 / L^{2}$. For this theory to be a consistent truncation of type IIB supergravity $[10,24,25]$ the Chern-Simons coupling $\kappa$ has to assume the value $1 / 2 \sqrt{3}$.

The action (2.1) leads to the equations of motion

$$
\begin{aligned}
G_{A B}-6 g_{A B}+2 F_{A C} F_{B}^{C}+\frac{1}{2} g_{A B} F_{C D} F^{C D} & =0, \\
\nabla_{B} F^{A B}+\kappa \epsilon^{A B C D E} F_{B C} F_{D E} & =0 .
\end{aligned}
$$

Static black hole solutions possessing spherical symmetry as well as their thermodynamics were discussed in detail in [10]. As shown there (following [26]) a scaling limit gives rise to 
the following solution:

$$
\begin{aligned}
d s^{2} & =-\frac{r^{2} f(r)}{L^{2}} d t^{2}+\frac{L^{2}}{r^{2} f(r)} d r^{2}+\frac{r^{2}}{L^{2}}\left(d x^{2}+d y^{2}+d z^{2}\right), \\
A_{t} & =h(r),
\end{aligned}
$$

where

$$
\begin{aligned}
& f(r)=\left(1-\frac{r_{0}^{2}}{r^{2}}\right)\left(1+\frac{r_{0}^{2}}{r^{2}}-\frac{q^{2}}{r_{0}^{2} r^{4}}\right), \\
& h(r)=\frac{\sqrt{3} q}{2 L}\left(\frac{1}{r_{0}^{2}}-\frac{1}{r^{2}}\right) .
\end{aligned}
$$

This solution possesses a planar event horizon. In consequence of AdS/CFT duality it describes the thermodynamics of plasma in equilibrium in flat Minkowski space.

The position of the (outer) event horizon is at $r=r_{0}$. There is also an inner horizon at $r=r_{-}$, where

$$
r_{-}^{2}=\frac{1}{2} r_{0}^{2}\left(\sqrt{1+4 \frac{q^{2}}{r_{0}^{6}}}-1\right) .
$$

Using standard Euclidean techniques one finds the Hawking temperature of the outer horizon $[21]$

$$
T=\frac{r_{0}}{\pi L^{2}}\left(1-\frac{q^{2}}{2 r_{0}^{6}}\right)
$$

Note that the temperature vanishes for the extremal black hole with $q^{2} / r_{0}^{6}=2$.

As discussed in [21] the chemical potential in the field theory is related to the asymptotic behaviour of the gauge field. It can be expressed in terms of $r_{0}$ and the Hawking temperature by

$$
r_{0}=\pi L^{2} \frac{T}{2}\left(1+\sqrt{1+\frac{2}{3} \frac{\mu^{2}}{T^{2}}}\right) .
$$

For the purposes of fluid-gravity duality it is appropriate to use coordinates which are not singular at the event horizon. The Schwarzschild-like coordinates used in [21] suffer from a coordinate singularity there. The choice made in [2] was to use EddingtonFinkelstein coordinates. Starting from the coordinates used above one can use a transformation of the form $r=r^{\prime}+F\left(r^{\prime}\right)$ to reach such a gauge. In the present case this results in the following expression of the charged black brane solution:

$$
\begin{aligned}
d s^{2} & =2 d r d t-\frac{r^{2} f(r)}{L^{2}} d t^{2}+\frac{r^{2}}{L^{2}}\left(d x^{2}+d y^{2}+d z^{2}\right), \\
A_{t} & =\frac{\sqrt{3} q}{2 L r^{2}}
\end{aligned}
$$

where the function $f$ is given above in eq. (2.4). 


\section{General form of the solution}

\subsection{The gradient expansion}

The method of [2] mimics the way relativistic hydrodynamics arises from the static, thermodynamic description. The energy-momentum tensor of perfect fluid hydrodynamics is just a boost of the equilibrium energy-momentum tensor, where the temperature and boost parameters are allowed to depend on position. Following the same idea one considers the boost of (2.9), which describes equilibrium states, to some constant velocity $u$, and then allows this velocity and the temperature to depend on $x$. The boost parameter $u^{\mu}$ is a 4 component velocity vector in the $x^{\mu}$ directions, normalized so that $u_{\mu} u^{\mu}=-1$ in the sense of the boundary metric $h_{\mu \nu}$ (metric on the conformal boundary of the locally asymptotically AdS spacetime (3.1)). Thus one is lead to consider the geometry ${ }^{3}$

$$
\mathrm{d} s^{2}=r^{2}\left(P_{\mu \nu}-2 B u_{\mu} u_{\nu}\right) \mathrm{d} x^{\mu} \mathrm{d} x^{\nu}-2 u_{\mu} \mathrm{d} x^{\mu} \mathrm{d} r,
$$

where ${ }^{4}$

$$
B=\frac{1}{2}\left(1-\frac{1}{b^{4} r^{4}}\left(1+q^{2} b^{6}\right)+\frac{q^{2}}{r^{6}}\right)
$$

and

$$
P_{\mu \nu}=h_{\mu \nu}+u_{\mu} u_{\nu}
$$

is the projector operator onto the space transverse to $u^{\mu}$, The vector potential takes the form:

$$
A=\frac{\sqrt{3} q}{2 r^{2}} u_{\mu} \mathrm{d} x^{\mu} .
$$

The constant parameter $b$ is just $1 / r_{0}$ in the notation of the previous section. The solution described there is recovered by going to the frame where $\left(u^{\mu}\right)=(1,0,0,0)$.

The geometry described above has a curvature singularity at $r=0$. The latter is shielded by the event horizon at $r=1 / b$. The parameter $b$ appearing in (3.1) is related to the Hawking temperature $T$ of the event horizon by eq. (2.7), which in the notation introduced above reads

$$
T=\frac{1}{2 \pi b}\left(2-q^{2} b^{6}\right) .
$$

The lines of constant $x^{\mu}$ in (3.1) are ingoing null geodesic, for large $r$ propagating in the direction set by $u^{\mu}$, and the radial coordinate $r$ parameterizes them in an affine way [13]. Unlike black holes in asymptotically flat spacetime, the metric (3.1) supports perturbations varying much slower within the transverse planes than within the radial direction. The parameter controlling the scale of variations in the radial direction is $b$.

The field configuration described above is a solution of the equations of motion for constant $b, q, u^{\mu}$. If these parameters are allowed to depend on $x$, the equations are violated by terms proportional to gradients of $b, q, u$. To cancel these, so as to ensure that the fields still satisfy Einstein equations, corrections need to be added to the metric and gauge potential order by order in an expansion in the number of gradients. Thus, if $b, u^{\mu}$ and

\footnotetext{
${ }^{3}$ From now on the constant $L$ is set to unity, and the notation is chosen to resemble that of reference [7].

${ }^{4}$ The notation is chosen so that in uncharged limit $(q \rightarrow 0, \kappa \rightarrow 0) B$ is equal to $B(b r)$ as defined in [7].
} 
$h_{\mu \nu}$ are allowed to vary slowly compared to the scale set by $b$, the metric (3.1) should be an approximate solution of nonlinear Einstein's equations with corrections organized in an expansion in the number of gradients in the $x^{\mu}$ directions. As in the uncharged case [2], this turns out to be possible if and only if $b, q, u$ satisfy differential equations which can be interpreted as the equations of hydrodynamics [2].

Technically this can be done by considering an arbitrary point, say $x=0$, and expanding the slowly varying quantities in Taylor series

$$
\begin{aligned}
u^{\mu}(x) & =u^{\mu}(0)+\epsilon x^{\alpha} \partial_{\alpha} u^{\mu}(0)+\ldots \\
b(x) & =b(0)+\epsilon x^{\alpha} \partial_{\alpha} b(0)+\ldots \\
q(x) & =q(0)+\epsilon x^{\alpha} \partial_{\alpha} q(0)+\ldots
\end{aligned}
$$

Each derivative with respect to the "boundary coordinates" $x$ is tagged with a power of $\epsilon$ for power counting purposes, and $\epsilon$ is set to unity at the end of calculations.

The four-dimensional boundary metric components $h_{\mu \nu}$ are also expanded around $x=0$, assuming that at zeroth order $h_{\mu \nu}(0)=\eta_{\mu \nu}$. Moreover, to simplify computations it is very useful to adopt a locally geodesic coordinate system on the boundary, so that all first order derivatives of $h_{\mu \nu}$ vanish. Thus one has

$$
h_{\mu \nu}(x)=\eta_{\mu \nu}+\mathcal{O}\left(\epsilon^{2}\right) .
$$

In general, the second order derivatives cannot of course be set to zero in this way, but these contributions are guaranteed to be tensorial and so the final results obtained are covariant in the boundary sense.

\subsection{Weyl covariance}

Weyl covariance in the bulk arises as an extension of the conformal symmetry of $\mathrm{N}=4$ supersymmetric Yang-Mills theory [7, 12]. A beautiful formalism allowing for manifest Weyl covariance in conformal hydrodynamics was introduced by Loganayagam [12] and applied to fluid-gravity duality in $[6,7]$.

The computation of the gradient expansion is simplified considerably by adopting the approach of [7], which imposes at the outset the conditions of Weyl invariance on the possible form of the solution. Conformal symmetry of the dual field theory can be extended to the bulk as follows $[6,7]$ :

$$
g_{\mu \nu} \rightarrow e^{-2 \phi} g_{\mu \nu}, \quad u^{\mu} \rightarrow e^{\phi} u^{\mu}, \quad b \rightarrow e^{-\phi} b \quad \text { and } \quad r \rightarrow e^{\phi} r
$$

where $\phi$ depends on the coordinates $x^{\mu}[7]$. A quantity which transforms homogeneously with a factor of $e^{w \phi}$ is said to transform with Weyl weight $w$. The Weyl weights of objects appearing in this paper are listed in appendix B.

The leading order metric (3.1) is Weyl-invariant, but due to the presence of $\mathrm{d} r$ it does not retain its form at higher orders. It can however be written in a manifestly Weylinvariant form upon introducing a vector field $\mathcal{A}_{\nu}$ defined by [12]

$$
\mathcal{A}_{\nu} \equiv u^{\lambda} \nabla_{\lambda} u_{\nu}-\frac{\nabla_{\lambda} u^{\lambda}}{3} u_{\nu}
$$


This quantity is of order one in the gradient expansion and transforms as a connection under Weyl-transformations

$$
\mathcal{A}_{\nu} \rightarrow \mathcal{A}_{\nu}+\partial_{\nu} \phi
$$

A powerful tool for generating Weyl-covariant gradient terms is the Weyl-covariant derivative $\mathcal{D}_{\mu}$, which uses the connection $\mathcal{A}_{\mu}$ (3.9) to compensate for derivatives of the Weyl factor coming from derivatives of Weyl-covariant tensors. It has the property that a Weylcovariant derivative of a Weyl-covariant expression is itself Weyl-covariant with the same weight $[7,12,13]$.

The manifestly Weyl-invariant form of the metric (3.1) reads

$$
\mathrm{d} s^{2}=\left(r^{2} P_{\mu \nu}-2 r^{2} B u_{\mu} u_{\nu}\right) \mathrm{d} x^{\mu} \mathrm{d} x^{\nu}-2 u_{\mu} \mathrm{d} x^{\mu}\left(\mathrm{d} r+r \mathcal{A}_{\nu} \mathrm{d} x^{\nu}\right)
$$

The correction involving $\mathcal{A}$ compensates an inhomogenous term in the transformation of $d r$. It is of first order in gradients; further contributions are needed for a complete solution at this order, but they are by themselves Weyl-invariant.

The static metric (3.1) is a leading order approximation to a spacetime whose metric is of the form [7]

$$
\mathrm{d} s^{2}=\left(\mathcal{G}_{\mu \nu}-2 u_{\mu} \mathcal{V}_{\nu}\right) \mathrm{d} x^{\mu} \mathrm{d} x^{\nu}-2 u_{\mu} \mathrm{d} x^{\mu}\left(\mathrm{d} r+r \mathcal{A}_{\nu} \mathrm{d} x^{\nu}\right),
$$

with the condition $u^{\mu} \mathcal{G}_{\mu \nu}=0$ completely fixing the gauge freedom [7]. This implies a choice of gauge which is different than that of $[4,5]$. It is easy to see that lines of constant $x$ are geodesics (affinely parameterized by $r$ ), as in the case of the static metric.

The metric (3.12) is manifestly Weyl-invariant, provided that the functions $\mathcal{V}_{\mu}$ are of unit Weyl weight and $\mathcal{G}_{\mu \nu}$ are Weyl invariant. The simplest way to construct them is by summing individual tensorial contributions of appropriate Weyl weight order by order in the gradient expansion, multiplied by scalar functions of the Weyl-invariant combinations $b r$ and $b^{3} q$.

Note finally that the Maxwell gauge field $A$ is a vector field of Weyl weight zero. It will be taken in the gauge $A_{r}=0$.

\section{The solution order by order}

\subsection{Zeroth order}

The solution at leading order is just the boosted charged black brane solution (3.1). The metric is obviously of the form (3.12) with

$$
\begin{aligned}
\mathcal{V}_{\mu} & =r^{2} B u_{\mu} \\
\mathcal{G}_{\mu \nu} & =r^{2} P_{\mu \nu}
\end{aligned}
$$

(strictly speaking, to retain only leading order terms, the term involving the Weyl connection in (3.12) must also be dropped, as it is of first order). 


\subsection{First order}

To find the complete solution at first order one needs to classify the possible terms that may appear. At first order there are:

- no Weyl-invariant scalars

- one Weyl-invariant pseudovector:

$$
l_{\mu}=\epsilon_{\mu \nu \lambda \rho} u^{\nu} \mathcal{D}^{\lambda} u^{\rho}
$$

- one Weyl-invariant vector:

$$
V_{0 \mu}=q^{-1} P_{\mu}^{\nu} \mathcal{D}_{\nu} q
$$

- one Weyl-invariant symmetric tensor ${ }^{5}$ of weight $w=-1$ :

$$
\sigma_{\mu \nu}=\frac{1}{2} \mathcal{D}_{(\mu} u_{\nu)}
$$

Therefore the general form of the Weyl-invariant structures appearing in the metric at this order is

$$
\begin{aligned}
\mathcal{V}_{\mu} & =r^{2} B u_{\mu}+r F_{1} l_{\mu}+b r^{2} F_{0} V_{0 \mu} \\
\mathcal{G}_{\mu \nu} & =r^{2} P_{\mu \nu}+2 b r^{2} F_{2} \sigma_{\mu \nu}
\end{aligned}
$$

where $F_{0}, F_{1}$ and $F_{2}$ are functions of the Weyl-invariants $r b$ and $b^{3} q$. These functions are to be determined by solving the field equations up to linear order in gradients. The factors of $b$ and $r$ above were chosen to ensure the correct Weyl weights (and partly also for convenience - the choice made above leads to a simple form of the differential equations).

Similarly, Weyl covariance implies that the vector potential takes the form:

$$
A=\left(\frac{\sqrt{3} q u_{\mu}}{2 r^{2}}+Y_{0} l_{\mu}+\tilde{Y}_{0} V_{0 \mu}\right) \mathrm{d} x^{\mu} .
$$

Once again, $Y_{0}$ and $\tilde{Y}_{0}$ are functions of $b r$ and $b^{3} q$ and will be determined by solving the equations of motion.

To find the unknown scalar functions $F_{0}, F_{1}, F_{2}, Y_{0}, \tilde{Y}_{0}$ the Ansatz described above is inserted into the field equations and expanded in gradients using (3.6). This process, while tedious (thus best relegated to symbolic manipulation software ${ }^{6}$ ), is not significantly more complicated than what has to be done to reproduce the results of [7].

As discussed in [2], the bulk field equations are of two types: the constraint equations, which impose consistency conditions on the slowly-varying background parameters $b(x), q(x), u^{\mu}(x)$ and the dynamical equations which determine the functions which appear in the field Ansatz (3.12).

\footnotetext{
${ }^{5}$ Symmetrization is defined as $A_{(\mu \nu)}:=A_{\mu \nu}+A_{\nu \mu}$.

${ }^{6}$ For most calculations in this paper Mathematica was used, but for some the Cadabra package [27, 28] was very convenient.
} 
The constraint equations take the form

$$
\begin{aligned}
& \partial_{0} q=-q \partial_{i} u_{i}, \quad\left(2-b^{6} q^{2}\right) \partial_{i} b-2 b\left(1+b^{6} q^{2}\right) \partial_{0} u_{i}=q b^{7} \partial_{i} q, \\
& \partial_{0} b=\frac{1}{3} b \partial_{i} u_{i} .
\end{aligned}
$$

In terms of the Weyl-covariant derivatives

$$
\begin{aligned}
& \mathcal{D}_{\mu} b=\partial_{\mu} b-\mathcal{A}_{\mu} b, \\
& \mathcal{D}_{\mu} q=\partial_{\mu} q+3 \mathcal{A}_{\mu} q,
\end{aligned}
$$

one can rewrite the constraints (4.7) as

$$
\begin{aligned}
\left(b^{6} q^{2}-2\right) \mathcal{D}_{\mu} b & =q b^{7} \mathcal{D}_{\mu} q, \\
u^{\mu} \mathcal{D}_{\mu} b & =0 .
\end{aligned}
$$

These equations can be interpreted as the equations of hydrodynamics at order zero, which describe the perfect fluid limit of the supersymmetric Yang-Mills plasma on the boundary. This will be discussed in more detail in section 5 below.

The remaining differential equations can be solved for the functions $F_{0}, F_{1}, F_{2}, Y_{0}, \tilde{Y}_{0}$. Taking into consideration the requirements: regularity across horizons and normalizability of the metric one can fix all constants of integration to find

$$
\begin{aligned}
F_{0}= & -\frac{1}{2 b r}+\frac{b^{6} q^{2}+2}{4 b^{4} r^{4}}-\frac{q^{2}\left(b^{6} q^{2}+2\right)}{4 r^{6}\left(b^{6} q^{2}+1\right)}+ \\
& +\frac{\left(b^{2} r^{2}-1\right)\left(-b^{6} q^{2}+b^{2} r^{2}+b^{4} r^{4}\right)}{2 b^{6} r^{6}} \int_{b r}^{\infty} \mathrm{d} x \frac{x^{4}\left(-b^{6} q^{2}(1+2 x)+x^{2}\left(3+2 x+x^{2}\right)\right)}{(1+x)^{2}\left(-b^{6} q^{2}+x^{2}+x^{4}\right)^{2}}, \\
F_{1}= & \frac{\sqrt{3} b^{4} \kappa q^{3}}{r^{5}\left(b^{6} q^{2}+1\right)}, \\
F_{2}= & \int_{b r}^{\infty} \mathrm{d} x \frac{x\left(1+x+x^{2}\right)}{(1+x)\left(-b^{6} q^{2}+x^{2}+x^{4}\right)}, \\
Y_{0}= & \frac{3 b^{4} \kappa q^{2}}{2 r^{2}\left(b^{6} q^{2}+1\right)}, \\
\tilde{Y}_{0}= & -\frac{\sqrt{3} b q\left(2+b^{6} q^{2}\right)}{8\left(1+b^{6} q^{2}\right) r^{2}}+ \\
& +\frac{\sqrt{3} b^{3} q}{2} \int_{b r}^{\infty} \frac{\mathrm{d} x}{x^{3}} \int_{x}^{\infty} \mathrm{d} y \frac{y^{4}\left(-b^{6} q^{2}(1+2 y)+y^{2}\left(3+2 y+y^{2}\right)\right)}{(1+y)^{2}\left(-b^{6} q^{2}+y^{2}+y^{4}\right)^{2}} .
\end{aligned}
$$

The symbols $b$ and $q$ appearing above are understood as values at an arbitrary point $x$, not necessarily $x=0$, which was just an irrelevant choice made to implement the gradient expansion. As stressed in the original papers on fluid-gravity duality [2], the equations are ultralocal in $x$, which is the key feature which allows one to determine the solution.

In the uncharged limit $(q \rightarrow 0, \kappa \rightarrow 0)$ one obtains

$$
F_{2}^{(q=0)}=\int_{b r}^{\infty} \frac{x^{3}-1}{x\left(x^{4}-1\right)} \mathrm{d} x,
$$

which is exactly the function $F(b r)$ appearing in the solution presented in [7]. In this limit the remaining functions vanish as expected. 


\subsection{Second order}

As in the previous section, the first step is to determine all the relevant Weyl-invariant structures which may appear at second order. In doing this one has to discard any terms which vanish or are not linearly independent after the first order constraints (4.9) are taken into account.

The results of this analysis are as follows:

- Scalars:

$$
\begin{aligned}
& S_{1}=b^{2} \sigma_{\mu \nu} \sigma^{\mu \nu}, \\
& S_{2}=b^{2} \omega_{\mu \nu} \omega^{\mu \nu}, \\
& S_{3}=b^{2} \mathcal{R}, \\
& S_{4}=b^{2} q^{-2} P^{\mu \nu} \mathcal{D}_{\mu} q \mathcal{D}_{\nu} q, \\
& S_{5}=b^{2} q^{-1} P^{\mu \nu} \mathcal{D}_{\mu} \mathcal{D}_{\nu} q, \\
& S_{6}=b^{2} q^{-1} P^{\mu \nu} l_{\mu} \mathcal{D}_{\nu} q
\end{aligned}
$$

- Vectors:

$$
\begin{aligned}
V_{1 \mu} & =b P_{\mu \nu} \mathcal{D}_{\rho} \sigma^{\nu \rho} \\
V_{2 \mu} & =b P_{\mu \nu} \mathcal{D}_{\rho} \omega^{\nu \rho}, \\
V_{3 \mu} & =b l^{\lambda} \sigma_{\mu \lambda}, \\
V_{4 \mu} & =b q^{-1} \sigma_{\mu}{ }^{\alpha} \mathcal{D}_{\alpha} q, \\
V_{5 \mu} & =b q^{-1} \omega_{\mu}{ }^{\alpha} \mathcal{D}_{\alpha} q,
\end{aligned}
$$

- Tensors:

$$
\begin{aligned}
T_{1 \mu \nu} & =u^{\rho} \mathcal{D}_{\rho} \sigma_{\mu \nu}, \\
T_{2 \mu \nu} & =C_{\mu \alpha \nu \beta} u^{\alpha} u^{\beta} \\
T_{3 \mu \nu} & =\omega_{\mu}^{\lambda} \sigma_{\lambda \nu}+\omega_{\nu}{ }^{\lambda} \sigma_{\lambda \mu}, \\
T_{4 \mu \nu} & =\sigma_{\mu}{ }^{\lambda} \sigma_{\lambda \nu}-\frac{1}{3} P_{\mu \nu} \sigma_{\alpha \beta} \sigma^{\mu \nu}, \\
T_{5 \mu \nu} & =\omega_{\mu}^{\lambda} \omega_{\lambda \nu}+\frac{1}{3} P_{\mu \nu} \omega_{\alpha \beta} \omega^{\alpha \beta}, \\
T_{6 \mu \nu} & =\Pi_{\mu \nu}^{\alpha \beta} \mathcal{D}_{\alpha} l_{\beta}, \\
T_{7 \mu \nu} & =\frac{1}{2} \epsilon^{\alpha \beta}{ }_{\lambda(\mu} C_{\alpha \beta \nu) \sigma} u^{\lambda} u^{\sigma}, \\
T_{8 \mu \nu} & =q^{-2} \Pi_{\mu \nu}^{\alpha \beta} \mathcal{D}_{\alpha} q \mathcal{D}_{\beta} q, \\
T_{9 \mu \nu} & =q^{-1} \Pi_{\mu \nu}^{\alpha \beta} \mathcal{D}_{\alpha} \mathcal{D}_{\beta} q, \\
T_{10 \mu \nu} & =q^{-1} \Pi_{\mu \nu}^{\alpha \beta} l_{\alpha} \mathcal{D}_{\beta} q, \\
T_{11 \mu \nu} & =\frac{1}{2} \epsilon_{(\mu}{ }^{\alpha \beta \lambda} \sigma_{\nu) \lambda} u_{\alpha} q^{-1} \mathcal{D}_{\beta} q .
\end{aligned}
$$


Here $\Pi_{\mu \nu}^{\alpha \beta}$ is the projector which can be used to create symmetric, traceless tensors:

$$
\Pi_{\mu \nu}^{\alpha \beta}=\frac{1}{2}\left(P_{\mu}^{\alpha} P_{\nu}^{\beta}+P_{\nu}^{\alpha} P_{\mu}^{\beta}-\frac{2}{3} P^{\alpha \beta} P_{\mu \nu}\right) .
$$

The scalar $\mathcal{R}$ is defined as in [7] and $C_{\mu \alpha \nu \beta}$ denotes the Weyl tensor. On the basis of the above results one can write down the most general form for the fields allowed by Weyl invariance:

$$
\begin{aligned}
\mathcal{V}_{\mu} & =r^{2} B u_{\mu}+r F_{1} l_{\mu}+b r^{2} F_{0} V_{0 \mu}+r^{2} \sum_{i=1}^{6} K_{i} S_{i} u_{\mu}+r \sum_{i=1}^{5} W_{i} V_{i \mu} \\
\mathcal{G}_{\mu \nu} & =r^{2} P_{\mu \nu}+2 b r^{2} F_{2} \sigma_{\mu \nu}+r^{2} \sum_{i=1}^{6} L_{i} S_{i} P_{\mu \nu}+\sum_{i=1}^{11} H_{i} T_{i \mu \nu} .
\end{aligned}
$$

The vector potential takes the form:

$$
A=\left(\frac{\sqrt{3} q u_{\mu}}{2 r^{2}}+Y_{0} l_{\mu}+\tilde{Y}_{0} V_{0 \mu}+r \sum_{i=1}^{6} N_{i} S_{i} u_{\mu}+\sum_{i=1}^{5} Y_{i} V_{i \mu}\right) \mathrm{d} x^{\mu} .
$$

The 39 coefficient functions $K_{i}, L_{i}, N_{i}(i=1, \ldots, 6), W_{i}, Y_{i}(i=1, \ldots, 5)$, and $H_{i}(i=$ $1, \ldots, 11)$ all depend on the Weyl invariant variables $b^{3} q$ and $b r$. Solving the equations of motion up to second order determines these functions uniquely. This procedure is more cumbersome than at first order, but as before one can simplify it by first establishing the constraints and the using them to simplify the remaining equations. In this way all the functions appearing in (3.12) are determined. The integration constants, as at first order, are all fixed by conditions of regularity, apart from the constants appearing in functions $K_{1}, \ldots, K_{6}, Y_{1}, \ldots, Y_{5}$, which can be fixed by choice of frame. The results are listed in appendix A.

Moreover, from the constraint equations one obtains relations involving second-order terms in $u_{\mu}, b$ and $q$. As in other similar contexts, these coincide with the equations of hydrodynamics at order one.

\section{Holography}

The holographic dictionary of the AdS/CFT correspondence provides a straightforward prescription for calculating physical quantities in the boundary theory. In practice, the computation involves subtraction of divergences, which is done in a systematic way using holographic renormalization $[29,30]$.

The expectation value of the energy momentum tensor is given by

$$
T_{\mu \nu}=-\frac{1}{8 \pi G_{N}} \lim _{r \rightarrow \infty} r^{2}\left(K_{\mu \nu}-K H_{\mu \nu}+3 H_{\mu \nu}-E_{\mu \nu}^{H}\right)
$$

where $G_{N}$ is the 5-dimensional Newton's constant, $H_{\mu \nu}$ is the induced metric at the surface $r=$ const and $E_{\mu \nu}^{H}$ is the corresponding Einstein tensor; $K_{\mu \nu}$ is the extrinsic curvature and 
$K$ is its trace. Carrying out the calculation requires a careful evaluation of the asymptotics of the integrals given in appendix A. One then finds the following result

$$
\begin{aligned}
T_{\mu \nu}= & \frac{1}{16 \pi G_{N}}\left(\frac{1+b^{6} q^{2}}{b^{4}}\left(P_{\mu \nu}+3 u_{\mu} u_{\nu}\right)-\frac{2 \sigma_{\mu \nu}}{b^{3}}+\frac{2\left(1+c_{1}\right) T_{1 \mu \nu}}{b^{2}}+\frac{2 T_{2 \mu \nu}}{b^{2}}+\frac{2 c_{1} T_{3 \mu \nu}}{b^{2}}+\right. \\
& +\frac{2 T_{4 \mu \nu}}{b^{2}}+\frac{4 b^{4} q^{2}\left(-1+b^{6} q^{2}\left(12 \kappa^{2}-1\right)\right)}{1+b^{6} q^{2}} T_{5 \mu \nu}+\frac{2 \sqrt{3} b^{7} q^{3} \kappa}{1+b^{6} q^{2}} T_{6 \mu \nu}+\frac{c_{8}}{b^{2}} T_{8 \mu \nu}+ \\
& \left.+\frac{c_{9}}{b^{2}} T_{9 \mu \nu}+\frac{c_{10}}{b^{2}} T_{10 \mu \nu}\right)
\end{aligned}
$$

where

$$
\begin{aligned}
& c_{1}=-\frac{b^{6} q^{2}+1}{2} \int_{1}^{\infty} \frac{\mathrm{d} x}{-b^{6} q^{2}+x+x^{2}}, \\
& c_{8}=\int_{1}^{\infty} \mathrm{d} x p_{8}(x), \quad c_{9}=\int_{1}^{\infty} \mathrm{d} x p_{9}(x), \quad c_{10}=\int_{1}^{\infty} \mathrm{d} x p_{10}(x),
\end{aligned}
$$

with $p_{8}(x), p_{9}(x), p_{10}(x)$ are defined in the appendix A (together with the second order solutions).

The result (5.2) agrees with [7] (for $d=4$ ) in the limit $q \rightarrow 0, \kappa \rightarrow 0$. From it one can read off the values of the transport coefficients.

This formula (5.2) shows that the equations of state are $\epsilon=3 p$ (as expected for a conformal theory) and

$$
\epsilon=\frac{3}{16 \pi G_{N}}\left(\frac{1}{b^{4}}+b^{2} q^{2}\right)
$$

(which is also consistent with conformal symmetry). This formula shows an interesting duality property, that is, invariance under the substitution

$$
\begin{aligned}
& b^{2} \longrightarrow \frac{1}{b q}, \\
& q^{2} \longrightarrow \frac{q}{b^{3}}
\end{aligned}
$$

Under this transformation the Weyl invariant quantity $b^{3} q$ is inverted:

$$
b^{3} q \longrightarrow \frac{1}{b^{3} q}
$$

This duality can be easily expressed in terms of $T$ and $\mu$.

Holographic renormalization of the current ${ }^{7}$ proceeds according to the formula [4]

$$
J_{\mu}=\lim _{r \rightarrow \infty} \frac{r^{2} A_{\mu}}{8 \pi G_{N}} .
$$

Inserting the solutions one finds:

$$
J_{\mu}=\frac{1}{8 \pi G_{N}}\left(\frac{\sqrt{3} q u_{\mu}}{2}+\frac{3 b^{4} q^{2} \kappa l_{\mu}}{2\left(1+b^{6} q^{2}\right)}-\frac{\sqrt{3} b^{3} q\left(2+b^{6} q^{2}\right)}{8\left(1+b^{6} q^{2}\right) b^{2}} V_{0 \mu}+\frac{3 \sqrt{3} b q}{8\left(1+b^{6} q^{2}\right)} V_{1 \mu}+\right.
$$

\footnotetext{
${ }^{7}$ The physical current, whose time component defines the density, differs from this by a factor of $2 \pi$, due to the normalization of the chemical potential adopted earler.
} 


$$
\begin{aligned}
& +\frac{3 \sqrt{3} b^{7} q^{3} \kappa^{2}}{\left(1+b^{6} q^{2}\right)^{2}} V_{2 \mu}-\frac{3 b^{4} \kappa q^{2}}{2\left(b^{6} q^{2}+1\right)^{2}} V_{3 \mu}+\frac{2 a_{4}\left(b^{6} q^{2}+1\right)+\sqrt{3} b^{9} q^{3}}{16 b^{2}\left(b^{6} q^{2}+1\right)^{2}} V_{4 \mu}+ \\
& \left.+\frac{a_{5}\left(b^{6} q^{2}+1\right)+\sqrt{3} b^{9}\left(24 \kappa^{2}-1\right) q^{3}-\sqrt{3} b^{3} q}{8 b^{2}\left(b^{6} q^{2}+1\right)^{2}} V_{5 \mu}\right)
\end{aligned}
$$

The constants $a_{4}$ and $a_{5}$ are

$$
\begin{aligned}
& a_{4}:=\int_{1}^{\infty} \mathrm{d} x \frac{2 \sqrt{3} b^{3} q\left(b^{12} q^{4}\left(3-2 x^{3}\right)+b^{6} q^{2} x^{2}\left(-3 x^{2}+2 x-3\right)+4 x^{3}\right)}{x^{2}\left(b^{6} q^{2}-2\right)\left(b^{6} q^{2}-x^{2}\left(x^{2}+1\right)\right)} \\
& a_{5}:=-\frac{\sqrt{3} b^{3} q\left(b^{12} q^{4}-3 b^{6} q^{2}+2\right)}{2 b^{6} q^{2}+2}+\int_{1}^{\infty} \mathrm{d} x \frac{3 \sqrt{3} b^{3} q F_{0}\left(b^{6} q^{2}\left(5 x^{2}-9\right)+x^{2}\left(3 x^{4}+5\right)\right)}{x^{4}} .
\end{aligned}
$$

This formula contains the values of the charge transport coefficients.

\section{Causal structure of the dual geometry}

This section is devoted to locating the event horizon in the geometry discussed above. It is important to verify that the metric singularity at $r=0$ is shielded by an event horizon, as expected on general grounds. As explained in [13] (see also $[18,19]$ ) is possible to find the event horizon in the gradient expansion by taking advantage of the fact that at zeroth order its location is known.

To determine the event horizon it is simplest to assume that it can be presented as the level set of a scalar function $S(r, x)$. This function must be Weyl-invariant and can be written in the gradient expansion in terms of all the independent scalars available up to some order. Since the event horizon at order zero is at $r=1 / b$, one has

$$
S(r, x)=b(x) r-g(x)
$$

where $g(x)$ is a Weyl-invariant function expanded in gradients of $b, q$ :

$$
g(x)=g_{0}(x)+g_{1}(x)+g_{2}(x)+\ldots
$$

Here $g_{k}$ denotes a linear combination of all Weyl-invariant scalars at order $k$ in the gradient expansion. Thus $g_{0}(x)$ is a function of Weyl weight zero, i.e. it depends only on the combination $b^{3} q$. There are no Weyl-invariant scalars a order 1 , and 6 at order 2 , so one expects to find

$$
\begin{aligned}
& g_{0}(x)=\lambda\left(b^{3} q\right) \\
& g_{1}(x)=0 \\
& g_{2}(x)=\sum_{k=1}^{6} h_{k}\left(b^{3} q\right) S_{k},
\end{aligned}
$$

where the $S_{i}$ are the 6 independent Weyl-invariant scalars (4.12). The functions $h_{i}$ and $\lambda$ will be determined in due course. Once this is done, the expression for the position of the event horizon will take the form

$$
r_{H}=\frac{1}{b}\left(\lambda+\sum_{k=1}^{6} h_{k} S_{k}\right) .
$$


The normal covector to a surface of the form (6.1) is

$$
m=d S
$$

which up to second order in the gradient expansion is

$$
m=r d b+b d r+d \lambda
$$

It is convenient to write the normal in terms of the Weyl-covariant derivatives (given in (4.8), (4.8)). One then has ${ }^{8}$

$$
m=\left(r \mathcal{D}_{\mu} b+\lambda^{\prime}\left(b^{3} \mathcal{D}_{\mu} q+3 q b^{2} \mathcal{D}_{\mu} b\right)\right) d x^{\mu}+b\left(d r+r \mathcal{A}_{\mu} d x^{\mu}\right) .
$$

Since the event horizon is a null surface this normal must satisfy $m^{2}=0$. At leading order this condition has two solutions: $\lambda=1$, which is the outer event horizon at order zero, and $\lambda=r_{-}$, where $r_{-}$is given in (2.6), which is the inner horizon of the charged black brane. At order two the condition fixes the functions $h_{k}$ appearing in (6.4). Carrying out this calculation with the metric obtained earlier one finds the outer event horizon at

$$
\begin{aligned}
r= & \frac{1}{b}+\frac{3\left(b^{6} q^{2}-2\right) K_{1}\left(b^{3} q, 1\right)+1}{3 b\left(b^{6} q^{2}-2\right)^{2}} S_{1}+\frac{2 b^{18}\left(12 \kappa^{2}-5\right) q^{6}+b^{12}\left(25-48 \kappa^{2}\right) q^{4}+80 b^{6} q^{2}+45}{60 b\left(b^{6} q^{2}-2\right)\left(b^{6} q^{2}+1\right)^{2}} S_{2}+ \\
& -\frac{1}{12 b\left(2-b^{6} q^{2}\right)} S_{3}+\left(\frac{K_{4}\left(b^{3} q, 1\right)}{b\left(b^{6} q^{2}-2\right)}+\frac{b^{11} q^{4}\left(17 b^{12} q^{4}+28 b^{6} q^{2}+20\right)}{32\left(b^{6} q^{2}-2\right)^{3}\left(b^{6} q^{2}+1\right)^{2}}\right) S_{4}+ \\
& +\left(\frac{K_{5}\left(b^{3} q, 1\right)}{b\left(b^{6} q^{2}-2\right)}-\frac{b^{5} q^{2}\left(b^{6} q^{2}+2\right)}{4\left(b^{18} q^{6}-3 b^{12} q^{4}+4\right)}\right) S_{5}+ \\
& +\frac{4\left(b^{18} q^{6}-3 b^{6} q^{2}-2\right) K_{6}\left(b^{3} q, 1\right)+\sqrt{3} b^{9} \kappa q^{3}\left(b^{6} q^{2}+4\right)\left(3 b^{6} q^{2}+2\right)}{4 b\left(-b^{12} q^{4}+b^{6} q^{2}+2\right)^{2}} S_{6} .
\end{aligned}
$$

It can be checked directly, by taking the $q \rightarrow 0$ limit, that the above formula reduces to the correct expression obtained in the uncharged case in [13] (see also [7, 19]).

Formally, the inner horizon at second order is given by

$$
r=r_{-}+h_{1} S_{1}+h_{2} S_{2}+h_{3} S_{3}+h_{4} S_{4}+h_{5} S_{5}+h_{6} S_{6},
$$

where the coefficient functions $h_{i}$ can be found in appendix C. As seen from the expressions given there, these functions (given in the form of integrals) diverge at $r=r_{-}$. This is not surprising, since the corrections to the metric and gauge field are divergent there already at first order, indicating a breakdown of the gradient expansion.

It may also seem interesting to consider the extremal limit, in which $r_{-}=1 / b$ so the inner and outer event horizons at zeroth order coincide. This corresponds to $b^{3} q=\sqrt{2}$. The gradient corrections to this horizon include divergent integrals, so one must conclude that the gradient construction breaks down in this extremal limit [5].

\footnotetext{
${ }^{8}$ Here $\lambda^{\prime} \equiv \lambda^{\prime}\left(b^{3} q\right)$.
} 


\section{Conclusions}

The main results of this article are the determination of the geometry dual to hydrodynamics with a conserved current in an arbitrary weakly curved background and the determination of its causal structure. The calculations of the bulk field theory solution in the gradient expansion described here follow the pattern of earlier calculations of this type and have benefited from a number of technical insights accumulated in this field. This made it possible to present the dual description in a form suitable for studying its causal structure following the method described in [13] (see also [18]). The location of the event horizon is found explicitly in section (6), thus showing that the locus $r=0$ is not a naked singularity. As is well known, the event horizon can also be used to define a hydrodynamic entropy current for the dual field theory [13].

Apart from the event horizon, the charged black brane geometry possesses also an inner horizon. The gradient corrections to the metric (as well as the gauge field) are singular at the location of the zeroth order inner horizon, indicating a breakdown of the gradient expansion. It is straightforward to formally determine the location of the inner horizon in the gradient expansion. The double integrals appearing in the formal expression for its location are divergent. This is reminiscent of the remarks in [5] concerning the extremal limit, in which the inner and outer horizons coincide. As noted in [5], the solution obtained in the gradient expansion develops singularities as the temperature $T$ vanishes with $q$ nonzero. This is apparently an indication that this limit is not described by hydrodynamics on the field theory side of the duality [31, 32]. It could however be that the extremal limit could be taken in such a way that an alternative gradient expansion would be valid.

An obvious extension of this work would be to determine the entropy current associated with the event horizon. This is left to a future work [20], where the entropy currents associated with dynamical horizons will also be discussed.

Finally, it would also be interesting to include background fields in this calculation, along the lines of $[8,22,23]$, where some important partial results are obtained.

\section{Acknowledgments}

The authors would like to thank Michał P. Heller for discussions and helpful comments on the manuscript, and Kasper Peeters for his excellent package Cadabra [27, 28]. This work was partially supported by Polish Ministry of Science and Higher Education grant N N202 173539.

\section{A Second order functions}

In the expressions below the prime denotes a partial derivative with respect to $b r$.

\section{A.1 Scalar sector}

$$
N_{1}=q \int_{b r}^{\infty} \mathrm{d} x\left(\frac{b^{2} x}{2 \sqrt{3} r}-\frac{\sqrt{3} x^{3}}{2 r^{3}}+\frac{x^{4}}{\sqrt{3} b r^{4}}\right) \frac{\left(1+x+x^{2}\right)^{2}}{(1+x)^{2}\left(-b^{6} q^{2}+x^{2}+x^{4}\right)^{2}},
$$




$$
\begin{aligned}
& N_{2}=-\frac{3 \sqrt{3} b^{6} \kappa^{2} q^{5}}{5 r^{9}\left(b^{6} q^{2}+1\right)^{2}}-\frac{\sqrt{3} q}{4 b^{2} r^{5}}-\frac{\sqrt{3} b^{2} \kappa^{2} q^{3}}{r^{7}\left(b^{6} q^{2}+1\right)} \\
& N_{3}=0 \text {, } \\
& N_{4}=\int_{b r}^{\infty} \mathrm{d} x\left(\frac{\sqrt{3} b^{5} F_{0} q^{3} x^{2}\left(b^{6} q^{2}(2 x-3)+4 x-3\right)(x-b r)^{2}(b r+2 x)}{r^{4}(x-1)^{2}(x+1)^{2}\left(b^{6} q^{2}+1\right)\left(-q^{2} b^{6}+x^{4}+x^{2}\right)^{2}}+\right. \\
& +\frac{2 \sqrt{3} F_{0}^{2} q x^{3}\left(b^{6} q^{2}\left(x^{2}-2\right)+x^{2}\right)(x-b r)^{2}(b r+2 x)}{b r^{4}(x-1)^{2}(x+1)^{2}\left(-q^{2} b^{6}+x^{4}+x^{2}\right)^{2}}+ \\
& -\frac{b^{5} q^{3} x(x-b r)^{2}(b r+2 x)}{8 \sqrt{3} r^{4}(x-1)^{2}(x+1)^{3}\left(b^{6} q^{2}+1\right)^{2}\left(-q^{2} b^{6}+x^{4}+x^{2}\right)^{3}}\left(2(x-1)^{2} x^{2}\right. \\
& \times\left(2 x^{4}+18 x^{3}+21 x^{2}+18 x+7\right)+b^{18} q^{6}\left(-4 x^{4}+2 x^{3}+9 x^{2}+x-14\right)+ \\
& +b^{12} q^{4}\left(4 x^{8}+10 x^{7}-17 x^{6}-3 x^{5}-15 x^{4}+15 x^{3}+35 x^{2}+17 x-28\right)+ \\
& \left.\left.+b^{6} q^{2}\left(8 x^{8}+32 x^{7}-49 x^{6}-21 x^{5}-33 x^{4}+15 x^{3}+34 x^{2}+16 x-14\right)\right)\right) \text {, } \\
& N_{5}=\int_{b r}^{\infty} \mathrm{d} x \frac{q x\left(b^{6} q^{2}(2 x+1)-x^{2}\left(x^{2}+2 x+3\right)\right)(x-b r)^{2}(b r+2 x)}{4 \sqrt{3} b r^{4}(x+1)^{2}\left(-q^{2} b^{6}+x^{4}+x^{2}\right)^{2}}, \\
& N_{6}=\int_{b r}^{\infty} \mathrm{d} x\left(-\frac{24 b^{8} F_{0} \kappa q^{4}(x-b r)^{2}(b r+2 x)}{r^{4}(x-1) x^{3}(x+1)\left(b^{6} q^{2}+1\right)\left(-q^{2} b^{6}+x^{4}+x^{2}\right)}+\right. \\
& -\frac{b^{2} \kappa q^{2}(x-b r)^{2}(b r+2 x)}{2 r^{4}(x-1) x^{4}(x+1)^{3}\left(b^{6} q^{2}+1\right)^{2}\left(-q^{2} b^{6}+x^{4}+x^{2}\right)^{3}}\left(b^{24} q^{8}\left(x^{5}+2 x^{4}+3 x^{2}+24 x+18\right)+\right. \\
& +b^{6} q^{2} x^{4}\left(-9 x^{9}-2 x^{8}-19 x^{7}-9 x^{6}+11 x^{5}+7 x^{4}+9 x^{3}+3 x^{2}+4 x+5\right) \\
& -2 x^{7}\left(3 x^{6}+2 x^{5}+x^{4}-3 x^{2}-2 x-1\right) \\
& +b^{18} q^{6}\left(2 x^{9}-4 x^{8}-7 x^{7}-18 x^{6}-48 x^{5}-45 x^{4}-54 x^{3}-48 x^{2}+12 x+18\right)+ \\
& +b^{12} q^{4} x^{2}\left(-3 x^{11}+2 x^{10}-5 x^{9}+15 x^{8}+43 x^{7}+47 x^{6}+60 x^{5}+57 x^{4}+15 x^{3}+\right. \\
& \left.\left.\left.+6 x^{2}-18 x-27\right)\right)\right) \\
& L_{1}=\frac{2}{3} F_{2}{ }^{2}-\frac{2}{3} \int_{b r}^{\infty} \frac{\mathrm{d} x}{x^{2}} \int_{x}^{\infty} \mathrm{d} y y^{2}\left(F_{2}{ }^{\prime}\right)^{2}, \\
& L_{2}=\frac{1}{3 b^{2} r^{2}}-\frac{4 b^{6} \kappa^{2} q^{4}}{5 r^{6}\left(b^{6} q^{2}+1\right)^{2}} \\
& L_{3}=0 \text {, } \\
& L_{4}=\int_{b r}^{\infty} \mathrm{d} x\left(-\frac{4 q x^{4}\left(b^{6} q^{2}+1\right)}{3(x-1)(x+1)\left(b^{6} q^{2}-2\right)\left(b^{6} q^{2}-x^{2}\left(x^{2}+1\right)\right)} \frac{\partial F_{0}}{\partial q}+\right. \\
& +\frac{4 F_{0}^{2} x^{5}\left(b^{6} q^{2}\left(2 x^{2}-3\right)+2 x^{2}\right)}{3\left(x^{2}-1\right)^{2}\left(-b^{6} q^{2}+x^{4}+x^{2}\right)^{2}}+\frac{F_{0} x^{4}}{3\left(x^{2}-1\right)^{2}\left(-b^{6} q^{2}+x^{2}+x^{4}\right)}\left(2 x^{2}\left(x^{4}-1\right)+\right. \\
& \left.+b^{18} q^{6}\left(7 x^{2}+6 x-15\right)+b^{12} q^{4}\left(x^{6}+12 x^{2}-5\right)+b^{6} q^{2}\left(3 x^{6}+3 x^{2}-24 x+10\right)\right)+ \\
& +\frac{b^{6} q^{2} x^{3}}{12\left(x^{2}-1\right)^{2}\left(b^{6} q^{2}-2\right)\left(b^{6} q^{2}+1\right)^{2}\left(b^{6} q^{2}-x^{2}\left(x^{2}+1\right)\right)^{2}}\left(b^{18} q^{6}\left(2 x^{3}-3 x^{2}+16 x-16\right)+\right. \\
& \left.-2 b^{12} q^{4}\left(x^{7}+3 x^{2}-22 x+16\right)+2 b^{6} q^{2}\left(x^{7}-3 x^{3}+6 x^{2}+2 x-8\right)+4 x\left(x^{6}-x^{2}+6 x-6\right)\right) \\
& \left.-\frac{2 x^{4} N_{4}{ }^{\prime \prime}}{3 \sqrt{3} b^{3} q}-\frac{10 x^{3} N_{4}{ }^{\prime}}{3 \sqrt{3} b^{3} q}-\frac{2 x^{2} N_{4}}{\sqrt{3} b^{3} q}\right) \\
& L_{5}=-\int_{b r}^{\infty} \mathrm{d} x\left(\frac{2 x^{4} F_{0}}{3\left(x^{2}-1\right)\left(-q^{2} b^{6}+x^{4}+x^{2}\right)}+\frac{x^{3}\left(b^{6} q^{2}(x-2)+2(x-1)\right)}{6\left(x^{2}-1\right)\left(b^{6} q^{2}+1\right)\left(b^{6} q^{2}-x^{2}\left(x^{2}+1\right)\right)}+\right. \\
& \left.+\frac{2 x^{4} N_{5}^{\prime \prime}}{3 \sqrt{3} b^{3} q}+\frac{10 x^{3} N_{5}^{\prime}}{3 \sqrt{3} b^{3} q}+\frac{2 x^{2} N_{5}}{\sqrt{3} b^{3} q}\right)
\end{aligned}
$$




$$
\begin{aligned}
& L_{6}=\int_{b r}^{\infty} \mathrm{d} x\left(\frac{8 \sqrt{3} b^{9} F_{0} \kappa q^{3}}{(x-1) x(x+1)\left(b^{6} q^{2}+1\right)\left(b^{6} q^{2}-x^{2}\left(x^{2}+1\right)\right)}-\frac{2 x^{4} N_{6}{ }^{\prime \prime}}{3 \sqrt{3} b^{3} q}-\frac{10 x^{3} N_{6}{ }^{\prime}}{3 \sqrt{3} b^{3} q}-\frac{2 x^{2} N_{6}}{\sqrt{3} b^{3} q}+\right. \\
& -\frac{b^{3} \kappa q}{\sqrt{3}(x-1) x^{2}(x+1)^{2}\left(b^{6} q^{2}+1\right)^{2}\left(b^{6} q^{2}-x^{2}\left(x^{2}+1\right)\right)^{2}}( \\
& +4 x^{5}\left(x^{6}+x^{5}+x^{4}-x^{2}-x-1\right)+b^{18} q^{6}\left(2 x^{4}+2 x^{3}+4 x^{2}-7 x-13\right)+ \\
& -b^{12} q^{4}\left(2 x^{8}+6 x^{7}+12 x^{6}-7 x^{5}-15 x^{4}-15 x^{3}-21 x^{2}+x+13\right)+ \\
& \left.\left.+b^{6} q^{2} x^{2}\left(4 x^{9}+4 x^{8}+4 x^{7}-2 x^{6}-10 x^{5}-22 x^{4}-3 x^{3}+7 x^{2}+7 x+11\right)\right)\right) \\
& K_{1}=-\frac{1}{2 b^{2} r^{2}}+\frac{1}{b^{4} r^{4}} \int_{b r}^{\infty} \mathrm{d} x\left(-x+\frac{x\left(1+x+2 x^{2}-b^{6} q^{2}(1+x)\right)}{6(1+x)\left(-b^{6} q^{2}+x^{2}+x^{4}\right)}+\right. \\
& \left.-\frac{2 b^{6} q^{2}}{x^{3}} \int_{x}^{\infty} \mathrm{d} y y\left(F_{2}{ }^{\prime}\right)^{2}-\frac{x^{2}-3 x^{6}+b^{6} q^{2}\left(x^{2}-6\right)}{3 x^{4}} \int_{x}^{\infty} \mathrm{d} y y^{2}\left(F_{2}{ }^{\prime}\right)^{2}\right) \\
& K_{2}=\frac{1}{2 b^{2} r^{2}}-\frac{6 b^{6} \kappa^{2} q^{6}}{5 r^{12}\left(b^{6} q^{2}+1\right)^{2}}-\frac{5 q^{2}}{12 b^{2} r^{8}}-\frac{7 b^{2} \kappa^{2} q^{4}}{5 r^{10}\left(b^{6} q^{2}+1\right)}+ \\
& +\frac{5 b^{18} q^{6}+3 b^{12}\left(4 \kappa^{2}+5\right) q^{4}+15 b^{6} q^{2}+5}{20 b^{6} r^{6}\left(b^{6} q^{2}+1\right)^{2}}, \\
& K_{3}=\frac{1}{12 b^{2} r^{2}} \\
& K_{4}=-\frac{b^{5} q^{2}}{3 r\left(4 b^{6} q^{2}+4\right)}+\frac{1}{b^{4} r^{4}} \int_{b r}^{\infty} \mathrm{d} x\left(\frac{-b^{6} q^{2} x}{48\left(x^{2}-1\right)^{2}\left(b^{6} q^{2}+1\right)^{2}\left(b^{6} q^{2}-x^{2}\left(x^{2}+1\right)\right)^{2}}(\right. \\
& +b^{18} q^{6}\left(8 x^{5}+3 x^{4}-20 x^{3}-2 x^{2}+12\right)+ \\
& -4 x^{2}\left(2 x^{7}+15 x^{6}-18 x^{5}+x^{4}-2 x^{3}-3 x^{2}+6 x-1\right)+ \\
& +b^{12} q^{4}\left(-8 x^{9}-15 x^{8}+36 x^{7}-4 x^{6}+24 x^{5}+15 x^{4}-64 x^{3}-12 x^{2}+24\right)+ \\
& \left.-4 b^{6} q^{2}\left(4 x^{9}+15 x^{8}-27 x^{7}+2 x^{6}-6 x^{5}-6 x^{4}+17 x^{3}+3 x^{2}-3\right)\right)+ \\
& +\frac{x^{4} F_{0}}{6\left(x^{2}-1\right)^{2}\left(b^{6} q^{2}+1\right)\left(-q^{2} b^{6}+x^{4}+x^{2}\right)^{2}}\left(b^{18} q^{6}\left(3 x^{2}+3 x-7\right)+\right. \\
& +b^{12} q^{4}\left(-12 x^{6}-9 x^{5}+21 x^{4}+9 x^{2}+9 x-14\right)+3\left(3 x^{10}-4 x^{6}+x^{2}\right)+ \\
& \left.+b^{6} q^{2}\left(9 x^{10}-24 x^{6}-18 x^{5}+21 x^{4}+9 x^{2}+6 x-7\right)\right)+ \\
& +\frac{F_{0}^{2} x^{5}\left(b^{12} q^{4}\left(2 x^{2}-3\right)+b^{6} q^{2}\left(-12 x^{6}+15 x^{4}+4 x^{2}-3\right)+2\left(3 x^{10}-6 x^{6}+x^{2}\right)\right)}{3\left(x^{2}-1\right)^{2}\left(-q^{2} b^{6}+x^{4}+x^{2}\right)^{2}}+ \\
& -\frac{x^{4}\left(b^{6} q^{2}-3 x^{4}+1\right) N_{4}{ }^{\prime \prime}}{3 \sqrt{3} b^{3} q}-\frac{x\left(b^{6} q^{2}\left(5 x^{2}+6\right)-15 x^{6}+5 x^{2}\right) N_{4}{ }^{\prime}}{3 \sqrt{3} b^{3} q} \\
& \left.-\frac{N_{4}\left(b^{6} q^{2}\left(x^{2}+2\right)-3 x^{6}+x^{2}\right)}{\sqrt{3} b^{3} q}\right) \\
& K_{5}=\frac{b^{6} q^{2}+2}{3 b r\left(4 b^{6} q^{2}+4\right)}+\frac{1}{b^{4} r^{4}} \int_{b r}^{\infty} \mathrm{d} x\left(\frac{x\left(x^{2}\left(3 x^{3}+3 x^{2}+3 x+1\right)-b^{6} q^{2}\left(x^{2}+2 x+2\right)\right)}{6(x+1)\left(-q^{2} b^{6}+x^{4}+x^{2}\right)}+\right. \\
& -\frac{x^{4}\left(b^{6} q^{2}-3 x^{4}+1\right) N_{5}{ }^{\prime \prime}}{3 \sqrt{3} b^{3} q}-\frac{x\left(b^{6} q^{2}\left(5 x^{2}+6\right)-15 x^{6}+5 x^{2}\right) N_{5}{ }^{\prime}}{3 \sqrt{3} b^{3} q}+ \\
& \left.-\frac{N_{5}\left(b^{6} q^{2}\left(x^{2}+2\right)-3 x^{6}+x^{2}\right)}{\sqrt{3} b^{3} q}\right) \\
& K_{6}=-\frac{2 b^{2} \kappa q}{\sqrt{3} r\left(b^{6} q^{2}+1\right)}+\frac{1}{b^{4} r^{4}} \int_{b r}^{\infty} \mathrm{d} x\left(\frac{-b^{3} \kappa q}{2 \sqrt{3}(x-1) x^{2}(x+1)^{2}\left(b^{6} q^{2}+1\right)^{2}\left(b^{6} q^{2}-x^{2}\left(x^{2}+1\right)\right)^{2}} .\right. \\
& \cdot\left(b^{24} q^{8}\left(2 x^{4}+2 x^{3}+x^{2}-4 x-7\right)+4 x^{5}\left(3 x^{7}+x^{6}+x^{5}+x^{4}-3 x^{3}-x^{2}-x-1\right)+\right. \\
& +b^{18} q^{6}\left(-8 x^{8}-6 x^{6}+10 x^{5}+35 x^{4}+14 x^{3}+10 x^{2}-5 x-14\right)+
\end{aligned}
$$




$$
\begin{aligned}
& +b^{6} q^{2} x^{2}\left(18 x^{10}+2 x^{9}+32 x^{8}+5 x^{7}-35 x^{6}-11 x^{5}-23 x^{4}-7 x^{3}+7 x^{2}+7 x+5\right)+ \\
& +b^{12} q^{4}\left(6 x^{12}-2 x^{11}+13 x^{10}-14 x^{9}-46 x^{8}-22 x^{7}+\right. \\
& \left.\left.-40 x^{6}-8 x^{5}+40 x^{4}+19 x^{3}+14 x^{2}-x-7\right)\right) \\
& +\frac{2 \sqrt{3} b^{9} \kappa q^{3} F_{0}\left(b^{6} q^{2}-5 x^{4}+1\right)}{x\left(x^{2}-1\right)\left(b^{6} q^{2}+1\right)\left(b^{6} q^{2}-x^{2}\left(x^{2}+1\right)\right)}-\frac{N_{6}\left(b^{6} q^{2}\left(x^{2}+2\right)-3 x^{6}+x^{2}\right)}{\sqrt{3} b^{3} q}+ \\
& \left.-\frac{x^{4}\left(b^{6} q^{2}-3 x^{4}+1\right) N_{6}^{\prime \prime}}{3 \sqrt{3} b^{3} q}-\frac{x\left(b^{6} q^{2}\left(5 x^{2}+6\right)-15 x^{6}+5 x^{2}\right) N_{6}{ }^{\prime}}{3 \sqrt{3} b^{3} q}\right)
\end{aligned}
$$

\section{A.2 Vector sector}

$$
\begin{aligned}
Y_{1}= & -\sqrt{3} b^{3} q \int_{b r}^{\infty} \frac{\mathrm{d} x}{x^{3}} \int_{x}^{\infty} \mathrm{d} y \frac{y^{6}(2+y)}{(1+y)^{2}\left(-b^{6} q^{2}+y^{2}+y^{4}\right)^{2}}+\frac{3 \sqrt{3} b q}{8\left(b^{6} q^{2}+1\right) r^{2}}, \\
Y_{2}= & \frac{3 \sqrt{3} b^{7} \kappa^{2} q^{3}}{\left(b^{6} q^{2}+1\right)^{2} r^{2}}, \\
Y_{3}= & \frac{3 b^{6} \kappa q^{2}}{b^{6} q^{2}+1} \int_{b r}^{\infty} \frac{\mathrm{d} x}{x^{3}} \int_{x}^{\infty} \mathrm{d} y \frac{y^{3}\left(b^{6} q^{2}(y+2)+3 y^{5}+6 y^{4}+9 y^{3}+6 y^{2}+4 y+2\right)}{(y+1)^{2}\left(-q^{2} b^{6}+y^{4}+y^{2}\right)^{2}}+ \\
& -\frac{3 b^{4} \kappa q^{2}}{2 r^{2}\left(b^{6} q^{2}+1\right)^{2}}, \\
Y_{4}= & -\int_{b r}^{\infty} \frac{\mathrm{d} x}{x^{3}} \int_{x}^{\infty} \mathrm{d} y \frac{y^{7}}{\left(1-y^{2}\right)^{2}\left(-b^{6} q^{2}+y^{2}+y^{4}\right)} \int_{1}^{y} \mathrm{~d} z \frac{2 \sqrt{3} b^{3} q(z-1)}{z^{2}\left(b^{6} q^{2}-2\right)\left(b^{6} q^{2}-z^{2}\left(z^{2}+1\right)\right)}( \\
& \left.3 b^{12} q^{4}\left(z^{2}+z+1\right)-b^{6} q^{2} z^{2}\left(z^{4}+z^{3}+2 z^{2}-z+3\right)+2 z^{3}\left(z^{3}+z^{2}+2 z+2\right)\right)+ \\
& +\frac{2 a_{4}\left(b^{6} q^{2}+1\right)+\sqrt{3} b^{9} q^{3}}{16 b^{2} r^{2}\left(b^{6} q^{2}+1\right)^{2}}, \\
Y_{5}= & \frac{\sqrt{3} b^{3} q}{b^{6} q^{2}+1} \int_{b r}^{\infty} \frac{\mathrm{d} x}{x^{3}} \int_{x}^{\infty} \mathrm{d} y \frac{y^{7}}{\left(1-y^{2}\right)^{2}\left(-b^{6} q^{2}+y^{2}+y^{4}\right)} \int_{1}^{y} \frac{\mathrm{d} z}{z^{5}}( \\
& b^{12} q^{4}\left(-2 z^{2}+9 z-10\right)+2 z^{2}\left(z^{4}-1\right)+2 b^{6} q^{2}\left(z^{6}-2 z^{2}+9 z-5\right)+ \\
& \left.+3 z F_{0}\left(b^{12} q^{4}\left(5 z^{2}-9\right)+b^{6} q^{2}\left(3 z^{6}+10 z^{2}-9\right)+z^{2}\left(3 z^{4}+5\right)\right)\right)+ \\
& +\frac{\sqrt{3} a_{5}\left(b^{6} q^{2}+1\right)+3 b^{9}\left(24 \kappa^{2}-1\right) q^{3}-3 b^{3} q}{8 \sqrt{3} b^{2} r^{2}\left(b^{6} q^{2}+1\right)^{2}}
\end{aligned}
$$

where

$$
\begin{aligned}
a_{4} & =\int_{1}^{\infty} \mathrm{d} x \frac{2 \sqrt{3} b^{3} q\left(b^{12} q^{4}\left(3-2 x^{3}\right)+b^{6} q^{2} x^{2}\left(-3 x^{2}+2 x-3\right)+4 x^{3}\right)}{x^{2}\left(b^{6} q^{2}-2\right)\left(b^{6} q^{2}-x^{2}\left(x^{2}+1\right)\right)}, \\
a_{5} & =-\frac{\sqrt{3} b^{3} q\left(b^{12} q^{4}-3 b^{6} q^{2}+2\right)}{2 b^{6} q^{2}+2}+\int_{1}^{\infty} \mathrm{d} x \frac{3 \sqrt{3} b^{3} q F_{0}\left(b^{6} q^{2}\left(5 x^{2}-9\right)+x^{2}\left(3 x^{4}+5\right)\right)}{x^{4}}, \\
W_{1}= & -b r \int_{b r}^{\infty} \mathrm{d} x\left(\frac{(x-1)(x+2)}{(x+1)\left(-q^{2} b^{6}+x^{2}+x^{4}\right)}+\right. \\
& \left.-\frac{2\left(b^{6} q^{2}\left(2 x^{2}-3\right)+2 x^{2}\right)}{x^{7}} \int_{x}^{\infty} \mathrm{d} y \frac{y^{6}(2+y)}{(1+y)^{2}\left(-b^{6} q^{2}+y^{2}+y^{4}\right)^{2}}\right)+ \\
& -\frac{3\left(b^{6} q^{2} r^{2}-b^{4} q^{2}+r^{2}\right)}{4 b^{3} r^{5}\left(b^{6} q^{2}+1\right)},
\end{aligned}
$$




$$
\begin{aligned}
& W_{2}=-\frac{1}{2 b r}+\frac{6 b^{7} \kappa^{2} q^{4}}{r^{5}\left(b^{6} q^{2}+1\right)^{2}}, \\
& W_{3}=b r \int_{b r}^{\infty} \mathrm{d} x\left(-\frac{\left(b^{6} q^{2} r^{2}-b^{4}\left(q^{2}+r^{6}\right)+r^{2}\right) Y_{3}{ }^{\prime \prime}}{\sqrt{3} b^{4} q r^{3}}+\frac{\left(b^{6} q^{2} r^{2}-3 b^{4}\left(q^{2}-r^{6}\right)+r^{2}\right) Y_{3}{ }^{\prime}}{\sqrt{3} b^{5} q r^{4}}+\right. \\
& -\frac{\sqrt{3} \kappa q\left(b\left(b r^{2}\left(3 b^{6} q^{2}+2\right)+r\left(3 b^{6} q^{2}+2\right)+2 b^{5} q^{2}-3 b^{5} r^{6}-3 b^{4} r^{5}-3 b^{3} r^{4}-b^{2} r^{3}\right)+2\right)}{b^{2} r^{3}\left(b^{6} q^{2}+1\right)(b r+1)\left(b^{4} q^{2}-b^{2} r^{4}-r^{2}\right)}+ \\
& \left.+\frac{8 \sqrt{3} F_{2} \kappa q}{b^{2} r^{5}}\right) \\
& W_{4}=b r \int_{b r}^{\infty} \mathrm{d} x\left(\frac{\left(b^{6} q^{2} r^{2}-3 b^{4}\left(q^{2}-r^{6}\right)+r^{2}\right) Y_{4}^{\prime}}{\sqrt{3} b^{5} q r^{4}}-\frac{(b r-1)(b r+1)\left(b^{4} q^{2}-b^{2} r^{4}-r^{2}\right) Y_{4}^{\prime \prime}}{\sqrt{3} b^{4} q r^{3}}+\right. \\
& \left.-\frac{2 b^{2} r^{4} F_{0}}{b^{6} q^{2} r^{2}-b^{4}\left(q^{2}+r^{6}\right)+r^{2}}-\frac{2 r^{3}\left(b^{8} q^{2}+b^{2}\right)-r\left(b^{6} q^{2}+2\right)}{2 b\left(b^{6} q^{2}+1\right)(b r-1)(b r+1)\left(b^{4} q^{2}-b^{2} r^{4}-r^{2}\right)}\right), \\
& W_{5}=b r \int_{b r}^{\infty} \mathrm{d} x\left(-\frac{\left(b^{2} r^{2}-1\right)\left(b^{4} q^{2}-b^{2} r^{4}-r^{2}\right) Y_{5}^{\prime \prime}}{\sqrt{3} b^{4} q r^{3}}+\frac{\left(b^{6} q^{2} r^{2}-3 b^{4}\left(q^{2}-r^{6}\right)+r^{2}\right) Y_{5}{ }^{\prime}}{\sqrt{3} b^{5} q r^{4}}+\right. \\
& +\frac{1}{2 b^{3} r^{5}\left(b^{6} q^{2}+1\right)\left(b^{2} r^{2}-1\right)\left(b^{4} q^{2}-b^{2} r^{4}-r^{2}\right)}\left(48 b^{4} \kappa^{2} q^{2}\left(b^{6} q^{2} r^{2}-b^{4}\left(q^{2}+r^{6}\right)+r^{2}\right)+\right. \\
& \left.+r^{2}\left(-2\left(b^{6} q^{2} r+r\right)^{2}-3 b^{5} q^{2} r\left(b^{6} q^{2}+2\right)+2 b^{4} r^{6}\left(b^{6} q^{2}+1\right)+6 b^{4} q^{2}\left(b^{6} q^{2}+1\right)\right)\right)+ \\
& \left.-\frac{F_{0}\left(5 b^{6} q^{2} r^{2}-b^{4}\left(7 q^{2}+r^{6}\right)+5 r^{2}\right)}{b^{8} q^{2} r^{4}-b^{6} r^{2}\left(q^{2}+r^{6}\right)+b^{2} r^{4}}\right)
\end{aligned}
$$

\section{A.3 Tensor sector}

$$
\begin{aligned}
H_{1}= & -(b r)^{2} \int_{b r}^{\infty} \mathrm{d} x \frac{x}{-b^{6} q^{2}+x^{2}+x^{4}}\left(1+\frac{1}{1-x^{2}} \int_{1}^{x} \mathrm{~d} y\left(6 y^{2} F_{2}+4 y^{3} F_{2}{ }^{\prime}\right)\right), \\
H_{2}= & -2(b r)^{2} \int_{b r}^{\infty} \mathrm{d} x \frac{x}{-b^{6} q^{2}+x^{2}+x^{4}}, \\
H_{3}= & (b r)^{2} \int_{b r}^{\infty} \mathrm{d} x \frac{x}{-b^{6} q^{2}+x^{2}+x^{4}}\left(1-\frac{1}{1-x^{2}} \int_{1}^{x} \mathrm{~d} y\left(6 y^{2} F_{2}+4 y^{3} F_{2}{ }^{\prime}\right)\right), \\
H_{4}= & 2(b r)^{2} F_{2}^{2}-2(b r)^{2} \int_{b r}^{\infty} \mathrm{d} x \frac{x}{-b^{6} q^{2}+x^{2}+x^{4}}, \\
H_{5}= & \frac{2(b r)^{2}}{\left(1+b^{6} q^{2}\right)^{2}} \int_{b r}^{\infty} \mathrm{d} x \frac{1}{x^{7}\left(-b^{6} q^{2}+x^{2}+x^{4}\right)}\left(-\left(1+b^{6} q^{2}\right)^{2} x^{4}\left(x^{2}+x^{4}+b^{6} q^{2}\left(1+2 x^{2}\right)\right)+\right. \\
& \left.+12 b^{12} \kappa^{2} q^{4}\left(b^{6} q^{2}\left(2\left(x^{6}+x^{4}+x^{2}\right)+3\right)+x^{2}\left(x^{2}-1\right)\left(2 x^{2}+1\right)\right)\right), \\
H_{6}= & \frac{2 \sqrt{3} b^{11} \kappa q^{3} r^{2}}{1+b^{6} q^{2}} \int_{b r}^{\infty} \mathrm{d} x \frac{x^{2}+x+1}{x^{2}(x+1)\left(-b^{6} q^{2}+x^{2}+x^{4}\right)}, \\
H_{7}= & 0, \\
H_{8}= & -(b r)^{2} \int_{b r}^{\infty} \mathrm{d} x \frac{x}{\left(1-x^{2}\right)\left(-b^{6} q^{2}+x^{2}+x^{4}\right)} \int_{1}^{x} \mathrm{~d} y p_{8}(y), \\
H_{9}= & -(b r)^{2} \int_{b r}^{\infty} \mathrm{d} x \frac{x}{\left(1-x^{2}\right)\left(-b^{6} q^{2}+x^{2}+x^{4}\right)} \int_{1}^{x} \mathrm{~d} y p_{9}(y), \\
H_{10}= & -(b r)^{2} \int_{b r}^{\infty} \mathrm{d} x \frac{x}{\left(1-x^{2}\right)\left(-b^{6} q^{2}+x^{2}+x^{4}\right)} \int_{1}^{x} \mathrm{~d} y p_{10}(y), \\
H_{11}= & 0,
\end{aligned}
$$

where:

$$
p_{8}(x)=-\frac{4 x^{2}\left(b^{6} q^{2}+1\right)\left(b^{6} q^{2}\left(x^{2}-3\right)+3 x^{6}+x^{2}\right)}{\left(x^{2}-1\right)\left(b^{6} q^{2}-2\right)\left(b^{6} q^{2}-x^{2}\left(x^{2}+1\right)\right)} \frac{\partial F_{0}}{\partial q}+
$$




$$
\begin{aligned}
& +\frac{4 F_{0}^{2} x^{3}\left(b^{12} q^{4}\left(4 x^{4}-15 x^{2}+12\right)+b^{6} q^{2} x^{2}\left(3 x^{4}+8 x^{2}-15\right)+4 x^{4}\right)}{\left(x^{2}-1\right)^{2}\left(-q^{2} b^{6}+x^{4}+x^{2}\right)^{2}}+ \\
& +\frac{2 F_{0} x^{2}}{\left(x^{2}-1\right)^{2}\left(b^{6} q^{2}-2\right)\left(b^{6} q^{2}+1\right)\left(-q^{2} b^{6}+x^{4}+x^{2}\right)^{2}}\left(4 x^{8}+2 x^{4}+\right. \\
& +b^{24} q^{8}\left(2 x^{4}+9 x^{3}-22 x^{2}-12 x+24\right)+4 b^{6} q^{2} x^{2}\left(4 x^{6}+3 x^{5}-6 x^{4}+2 x^{2}-9 x+4\right) \\
& -6 x^{12}+b^{18} q^{6}\left(8 x^{8}-3 x^{7}-6 x^{6}+8 x^{4}+9 x^{3}-28 x^{2}+6\right)+ \\
& \left.+2 b^{12} q^{4}\left(3 x^{12}+10 x^{8}-15 x^{6}+6 x^{4}-18 x^{3}+5 x^{2}+24 x-9\right)\right) \\
& -\frac{b^{6} q^{2} x}{4\left(x^{2}-1\right)^{2}\left(b^{6} q^{2}-2\right)\left(b^{6} q^{2}+1\right)^{2}\left(b^{6} q^{2}-x^{2}\left(x^{2}+1\right)\right)^{2}}( \\
& b^{24} q^{8}\left(3 x^{4}-12 x^{3}+8 x^{2}+36 x-36\right)+8 x^{2}\left(3 x^{6}-6 x^{5}+x^{4}-3 x^{2}+6 x-1\right)+ \\
& +b^{18} q^{6}\left(-3 x^{8}-36 x^{7}+44 x^{6}+9 x^{4}-72 x^{3}+28 x^{2}+132 x-96\right)+ \\
& +4 b^{6} q^{2}\left(3 x^{8}-24 x^{7}+15 x^{6}-9 x^{4}+12 x^{3}+23 x^{2}-12 x-6\right)+ \\
& \left.-6 b^{12} q^{4}\left(x^{8}+14 x^{7}-16 x^{6}+x^{4}+10 x^{3}-14 x^{2}-8 x+14\right)\right) \\
p_{9}(x)= & \frac{x}{\left(x^{2}-1\right)\left(b^{6} q^{2}+1\right)\left(b^{6} q^{2}-x^{2}\left(x^{2}+1\right)\right)}\left(b^{6} q^{2}\left(b^{6} q^{2}(3 x-4)+6 x-4\right)+\right. \\
& \left.+2 x F_{0}\left(b^{12} q^{4}\left(x^{2}-3\right)+b^{6} q^{2}\left(3 x^{6}+2 x^{2}-3\right)+3 x^{6}+x^{2}\right)\right) \\
& \left.+4 x F_{0}\left(b^{12} q^{4}\left(3 x^{2}-4\right)-b^{6} q^{2}\left(x^{6}-6 x^{2}+4\right)-x^{2}\left(x^{4}-3\right)\right)\right) \\
p_{10}(x)= & \frac{-x^{3}}{x^{4}\left(x^{2}-1\right)\left(b^{6} q^{2}+1\right)^{2}\left(-q^{2} b^{6}+x^{4}+x^{2}\right)}\left(x^{2}\left(2 x^{5}-3 x^{4}-2 x+3\right)+\right. \\
& +b^{6} q^{2}\left(x^{7}-3 x^{6}-3 x^{3}+6 x^{2}+8 x-7\right)-b^{12} q^{4}\left(x^{3}-3 x^{2}-4 x+7\right)+ \\
& +4 x
\end{aligned}
$$

The existence of terms $1 /\left(1-x^{2}\right)$ in $H_{1}, H_{3}, H_{4}$ may suggest that there is a problem at the outer horizon. However, examining the near-horizon behaviour one can verify that each of these functions is regular. To see this one must make use of the explicit form of $F_{2}$.

\section{B Weyl weights}

Weight $4:$ tensors from $T_{1}^{\mu \nu}$ to $T_{11}^{\mu \nu}$,

Weight $\quad 3: \quad q, \sigma^{\mu \nu}, \omega^{\mu \nu}$

Weight $2: g^{\mu \nu}, l^{\mu}, V_{0}{ }^{\mu}$, vectors from $V_{1}^{\mu}$ to $V_{5}^{\mu}$,

Weight $\quad 1: T, \mu, u^{\mu}, r$,

Weight $\quad 0: l_{\mu}, V_{0 \mu}$, Weyl-invariant scalars, all covariant vectors and tensors

Weight $-1: \quad b, u_{\mu}, \sigma_{\mu \nu}, \omega_{\mu \nu}$.

Weight $-2: g_{\mu \nu}$.

\section{Inner horizon at second order}

The location of the inner horizon is given by (6.9). The coefficient functions appearing there are given by

$$
\begin{aligned}
h_{1} & =\frac{b^{4} r_{-}^{5} K_{1}\left(b r_{-} \sqrt{b^{2} r_{-}^{2}+1}, b r_{-}\right)}{-2 b^{4} r_{-}^{4}+b^{2} r_{-}^{2}+1}-\frac{b^{3} r_{-}^{4}\left(5 b^{2} r_{-}^{2}+4\right)}{3\left(b^{2} r_{-}^{2}+2\right)\left(-2 b^{4} r_{-}^{4}+b^{2} r_{-}^{2}+1\right)^{2}}, \\
h_{2} & =\frac{1}{60 b^{2} r_{-}}\left(\frac{24 \kappa^{2}\left(b^{2} r_{-}^{2}+1\right)^{2}}{\left(b^{4} r_{-}^{4}+b^{2} r_{-}^{2}+1\right)^{2}}+\frac{5\left(-9 b^{4} r_{-}^{4}+2 b^{2} r_{-}^{2}+2\right)}{2 b^{4} r_{-}^{4}-b^{2} r_{-}^{2}-1}\right),
\end{aligned}
$$




$$
\begin{aligned}
h_{3}= & \frac{b^{2} r_{-}^{3}}{12\left(-2 b^{4} r_{-}^{4}+b^{2} r_{-}^{2}+1\right)}, \\
h_{4}= & -\frac{r_{-}^{3}\left(b^{3} r_{-}^{2}+b\right)^{2}}{4\left(b^{2} r_{-}^{2}+2\right)^{2}\left(b^{4} r_{-}^{4}+b^{2} r_{-}^{2}+1\right)\left(2 b^{4} r_{-}^{4}-b^{2} r_{-}^{2}-1\right)^{3}}\left(-12 b^{5} r_{-}^{5}+82 b^{4} r_{-}^{4}-4 b^{3} r_{-}^{3}+\right. \\
& +32 b^{2} r_{-}^{2}+8+8 b^{13} r_{-}^{13}+32 b^{12} r_{-}^{12}+18 b^{11} r_{-}^{11}+80 b^{10} r_{-}^{10}+b^{9} r_{-}^{9}+130 b^{8} r_{-}^{8}-11 b^{7} r_{-}^{7}+ \\
& \left.+122 b^{6} r_{-}^{6}\right)+\frac{b^{4} r_{-}^{5} F_{0}^{2}\left(b r_{-} \sqrt{b^{2} r_{-}^{2}+1}, b r_{-}\right)}{-4 b^{4} r_{-}^{4}+2 b^{2} r_{-}^{2}+2}+\frac{b^{4} r_{-}^{5} K_{4}\left(b r_{-} \sqrt{b^{2} r_{-}^{2}+1}, b r_{-}\right)}{-2 b^{4} r_{-}^{4}+b^{2} r_{-}^{2}+1}+ \\
& -\frac{b^{3} r_{-}^{4}\left(4 b^{6} r_{-}^{6}+7 b^{4} r_{-}^{4}+5 b^{2} r_{-}^{2}+2\right) F_{0}\left(b r_{-} \sqrt{b^{2} r_{-}^{2}+1}, b r_{-}\right)}{\left(b^{2} r_{-}^{2}+2\right)\left(-2 b^{4} r_{-}^{4}+b^{2} r_{-}^{2}+1\right)^{2}} \\
h_{5}= & \frac{b^{4} r_{-}^{5} K_{5}\left(b r_{-} \sqrt{b^{2} r_{-}^{2}+1}, b r_{-}\right)}{-2 b^{4} r_{-}^{4}+b^{2} r_{-}^{2}+1}+\frac{b^{3} r_{-}^{4}\left(4 b^{10} r_{-}^{10}+11 b^{8} r_{-}^{8}+20 b^{6} r_{-}^{6}+21 b^{4} r_{-}^{4}+12 b^{2} r_{-}^{2}+4\right)}{4\left(-2 b^{4} r_{-}^{4}+b^{2} r_{-}^{2}+1\right)^{2}\left(b^{6} r_{-}^{6}+3 b^{4} r_{-}^{4}+3 b^{2} r_{-}^{2}+2\right)} \\
h_{6}= & \frac{b^{4} r_{-}^{5} K_{6}\left(b r_{-} \sqrt{\left.b^{2} r_{-}^{2}+1, b r_{-}\right)}\right.}{-2 b^{4} r_{-}^{4}+b^{2} r_{-}^{2}+1}+ \\
& -\frac{\sqrt{3} \kappa r_{-}\left(b^{2} r_{-}^{2}+1\right)^{3 / 2}\left(2 b^{4} r_{-}^{4}+b^{2} r_{-}^{2}+1\right)\left(4 b^{4} r_{-}^{4}+3 b^{2} r_{-}^{2}+2\right)}{\left(-2 b^{4} r_{-}^{4}+b^{2} r_{-}^{2}+1\right)^{2}\left(b^{6} r_{-}^{6}+3 b^{4} r_{-}^{4}+3 b^{2} r_{-}^{2}+2\right)} \\
& -\frac{\sqrt{3} b \kappa r_{-}^{2}\left(b^{2} r_{-}^{2}+1\right)^{3 / 2} F_{0}\left(b r_{-} \sqrt{b^{2} r_{-}^{2}+1}, b r_{-}\right)}{2 b^{8} r_{-}^{8}+b^{6} r_{-}^{6}-2 b^{2} r_{-}^{2}-1} .
\end{aligned}
$$

In the last formula above, some functions, such as for example $K_{4}\left(b r_{-} \sqrt{b^{2} r_{-}^{2}+1}, b r_{-}\right)$, are singular. In fact at the inner horizon, starting from first order, metric and vector potential are also singular. For instance, the first order function $F_{2}$ can be rewritten as follows:

$$
\begin{aligned}
F_{2} & =\int_{b r}^{\infty} \mathrm{d} x \frac{x\left(1+x+x^{2}\right)}{(1+x)\left(-b^{6} q^{2}+x^{2}+x^{4}\right)} \\
& =\int_{b r}^{\infty} \mathrm{d} x \frac{x\left(1+x+x^{2}\right)}{(1+x)\left(x-b r_{-}\right)\left(x+b r_{-}\right)\left(1+b^{2} r_{-}^{2}+x^{2}\right)} .
\end{aligned}
$$

The last expression is singular in the limit $r \rightarrow r_{-}$, so $F_{2}$ diverges across the inner horizon. The same holds for many other second-order functions, like $K_{4}$ or $K_{5}$.

Open Access. This article is distributed under the terms of the Creative Commons Attribution License which permits any use, distribution and reproduction in any medium, provided the original author(s) and source are credited.

\section{References}

[1] J. Casalderrey-Solana, H. Liu, D. Mateos, K. Rajagopal and U.A. Wiedemann, Gauge/string duality, hot QCD and heavy ion collisions, arXiv:1101.0618 [INSPIRE].

[2] S. Bhattacharyya, V.E. Hubeny, S. Minwalla and M. Rangamani, Nonlinear fluid dynamics from gravity, JHEP 02 (2008) 045 [arXiv: 0712.2456] [INSPIRE].

[3] R.A. Janik and R.B. Peschanski, Asymptotic perfect fluid dynamics as a consequence of AdS/CFT, Phys. Rev. D 73 (2006) 045013 [hep-th/0512162] [INSPIRE].

[4] N. Banerjee et al., Hydrodynamics from charged black branes, JHEP 01 (2011) 094 [arXiv:0809.2596] [INSPIRE]. 
[5] J. Erdmenger, M. Haack, M. Kaminski and A. Yarom, Fluid dynamics of R-charged black holes, JHEP 01 (2009) 055 [arXiv:0809.2488] [INSPIRE].

[6] S. Bhattacharyya et al., Forced fluid dynamics from gravity, JHEP 02 (2009) 018 [arXiv:0806.0006] [INSPIRE].

[7] S. Bhattacharyya, R. Loganayagam, I. Mandal, S. Minwalla and A. Sharma, Conformal nonlinear fluid dynamics from gravity in arbitrary dimensions, JHEP 12 (2008) 116 [arXiv: 0809.4272] [INSPIRE].

[8] J. Hur, K.K. Kim and S.-J. Sin, Hydrodynamics with conserved current from the gravity dual, JHEP 03 (2009) 036 [arXiv: 0809.4541] [INSPIRE].

[9] T. Kalaydzhyan and I. Kirsch, Holographic dual of a boost-invariant plasma with chemical potential, JHEP 02 (2011) 053 [arXiv: 1012.1966] [INSPIRE].

[10] A. Chamblin, R. Emparan, C.V. Johnson and R.C. Myers, Charged AdS black holes and catastrophic holography, Phys. Rev. D 60 (1999) 064018 [hep-th/9902170] [INSPIRE].

[11] D.T. Son and P. Surowka, Hydrodynamics with triangle anomalies, Phys. Rev. Lett. 103 (2009) 191601 [arXiv:0906.5044] [INSPIRE].

[12] R. Loganayagam, Entropy current in conformal hydrodynamics, JHEP 05 (2008) 087 [arXiv:0801.3701] [INSPIRE].

[13] S. Bhattacharyya et al., Local fluid dynamical entropy from gravity, JHEP 06 (2008) 055 [arXiv:0803.2526] [INSPIRE].

[14] P. Romatschke, Relativistic viscous fluid dynamics and non-equilibrium entropy, Class. Quant. Grav. 27 (2010) 025006 [arXiv:0906.4787] [INSPIRE].

[15] K. Jensen et al., Towards hydrodynamics without an entropy current, Phys. Rev. Lett. 109 (2012) 101601 [arXiv:1203.3556] [INSPIRE].

[16] N. Banerjee et al., Constraints on fluid dynamics from equilibrium partition functions, JHEP 09 (2012) 046 [arXiv: 1203.3544] [INSPIRE].

[17] I. Booth, M.P. Heller and M. Spalinski, Black brane entropy and hydrodynamics: the Boost-invariant case, Phys. Rev. D 80 (2009) 126013 [arXiv:0910.0748] [INSPIRE].

[18] I. Booth, M.P. Heller and M. Spalinski, Black brane entropy and hydrodynamics, Phys. Rev. D 83 (2011) 061901 [arXiv:1010.6301] [InSPIRE].

[19] I. Booth, M.P. Heller, G. Plewa and M. Spalinski, On the apparent horizon in fluid-gravity duality, Phys. Rev. D 83 (2011) 106005 [arXiv:1102.2885] [INSPIRE].

[20] G. Plewa and M. Spalinski, Entropy currents from holography in hydrodynamics with charge, in preparation.

[21] R.C. Myers, M.F. Paulos and A. Sinha, Holographic hydrodynamics with a chemical potential, JHEP 06 (2009) 006 [arXiv:0903.2834] [inSPIRE].

[22] D.E. Kharzeev and H.-U. Yee, Anomalies and time reversal invariance in relativistic hydrodynamics: the second order and higher dimensional formulations, Phys. Rev. D 84 (2011) 045025 [arXiv:1105.6360] [INSPIRE].

[23] R. Loganayagam, Anomaly induced transport in arbitrary dimensions, arXiv:1106.0277 [INSPIRE]. 
[24] J.P. Gauntlett, E. O Colgain and O. Varela, Properties of some conformal field theories with M-theory duals, JHEP 02 (2007) 049 [hep-th/0611219] [INSPIRE].

[25] J.P. Gauntlett and O. Varela, Consistent Kaluza-Klein reductions for general supersymmetric AdS solutions, Phys. Rev. D 76 (2007) 126007 [arXiv:0707.2315] [INSPIRE].

[26] E. Witten, Anti-de Sitter space, thermal phase transition and confinement in gauge theories, Adv. Theor. Math. Phys. 2 (1998) 505 [hep-th/9803131] [InSPIRE].

[27] K. Peeters, A field-theory motivated approach to symbolic computer algebra, Comput. Phys. Commun. 176 (2007) 550 [cs/0608005] [inSPIRE].

[28] K. Peeters, Introducing Cadabra: a symbolic computer algebra system for field theory problems, hep-th/0701238 [INSPIRE].

[29] M. Henningson and K. Skenderis, The holographic Weyl anomaly, JHEP 07 (1998) 023 [hep-th/9806087] [INSPIRE].

[30] V. Balasubramanian and P. Kraus, A stress tensor for anti-de Sitter gravity, Commun. Math. Phys. 208 (1999) 413 [hep-th/9902121] [INSPIRE].

[31] S.S. Gubser and I. Mitra, Instability of charged black holes in Anti-de Sitter space, hep-th/0009126 [INSPIRE].

[32] S. Bhattacharyya, S. Lahiri, R. Loganayagam and S. Minwalla, Large rotating AdS black holes from fluid mechanics, JHEP 09 (2008) 054 [arXiv:0708.1770] [INSPIRE]. 